\title{
Contemporary Layout’s Integration for Geospatial Image Mining
}

\author{
Riaz Ahmed Shaikh \\ School of Computer Science and \\ Engineering, UESTC \\ Chengdu 611731, China
}

\author{
Jian-Ping Li \\ School of Computer Science and \\ Engineering, UESTC \\ Chengdu 611731, China
}

\author{
Asif Khan \\ School of Computer Science and \\ Engineering, UESTC \\ Chengdu 611731, China
}

\begin{abstract}
Image taxonomy and repossession plays a major role in dealing with large multimedia data on the Internet. Social networks, image sharing websites and mobile application require categorizing multimedia items for more efficient search and storage. Therefore, image classification and retrieval methods gained a great importance for researchers and companies. Image classification can be performed in a supervised and semisupervised manner and in order to categorize an unknown image, a statistical model created using relabeled samples is fed with the numerical representation of the visual features of images. Analysis of the keywords surrounding the images or the content of the images alone has not yet achieved results that would allow deriving precise location information to select representative images. Photos that are reliably tagged with labels of place names or areas only cover a small fraction of available images and also remain at a keyword level. State of the art of content based retrieval has been analyzed in earth perception image archives concentrating on complete frameworks indicating guarantee for the operational implementation. The methods are taken into consideration, concentrating specifically on the stages after extraction of primitive features. The solutions conceived for the issues such as synthesis and simplification of features, semantic labeling and indexing are reviewed. The approaches regarding query execution and specification are assessed where conclusions are drawn in the research of earth observation mining.
\end{abstract}

Keywords-Geo-Location; Spatial Layout; Feature Extraction; Image Mining

\section{INTRODUCTION}

Nowadays, the role of geographic location for images and the connection of web information to a physical location have getting significance attention. Web search is increasingly becoming "local", search engines not only index content by keywords but also by geographical locations. Users can have information mapped to a certain region-of-interest or place and search accordingly. Web image search, on the other hand, is mostly keyword-based or explores basic image features such as color or texture.

A geospatial Web image search that works on unstructured Web pages with arbitrary images is not yet available. Image search associated with a certain area rapidly demonstrates that Web pictures commonly don't effortlessly uncover their explicit connection to a real geographic position. However, geographic definitions of web pictures are not accessible at all or concealed some place inside of the content of Web page. This absence of explicit area data additionally applies to pictures contained in Web pages. Despite the fact that GPS has touched base at the consumer, the photographs that really have an area stamp or are geo-labeled cover just a minor portion of all Web pictures. Furthermore, such images are mostly only available in social communities, but seldom find their way into the interesting asset of local Web pages. This represents an intriguing challenge to the field of Web picture retrieval, to be specific, the determination of dependable information of location for Web pictures from disorganized Web pages.

Right now, a Web search photographs of famous place name remains a keyword based search of well-known geographic place names. This can work for known places, landmarks, or regions such as "London" or "Beijing” but cannot bear a precise geo-reference for Web image content. In this context there is need a spatial search of web image to facilitate clients to look for images from a certain geographical coordinate or its spatial neighborhood to complement geospatial Web search engines. In this work, a system for geospatial Web image search has been developed [1]. The aim of this study, at a general approach for common Web images that work beyond keyword search and do not need prepared tags or annotations.

Conceivable location information has been determined for those pictures which exist on the Web pages by a set up spatial connection. The common connection of a content of web page, area, its holds pictures, and related data about data means metadata is a fundamental main thrust behind this methodology. In this study spatial search engine has been utilized for a location-based geo-referenced search down to the address level [2]. It utilizes the information regarding location of a web-page, and it is not unequivocally hold as metadata or other organized annotation yet is fairly a verifiable piece of the textual content.

\section{GEO SPATIAL WEB SEARCH}

Querying for Web pages associated to a convinced given location has been allowed by geospatial search. The majority existing search engines are already most proficient for queries based on keywords, but lack geospatial capabilities since the search for geospatial information poses some special requirements that they cannot completely fulfill. For example, certain location-based queries cannot be answered with a purely textual index, such as vicinity queries within a certain radius, results in a specific district of a city, or other geospatial constraints. Furthermore, the result presentation should consider and cater for the geospatial dimension. 
Nowadays, location-based Web search and also the services working on prepared geo-referenced information getting very much attention. This is reliable with the high significance of area to the client. On the other hand, the Web contains a massive amount of resources that exhibit geospatial references. Web pages with the required information related with location include home pages of businesses, restaurants, agencies, museums etc. These are excellent sources to answer user's queries for relevant spatial information.

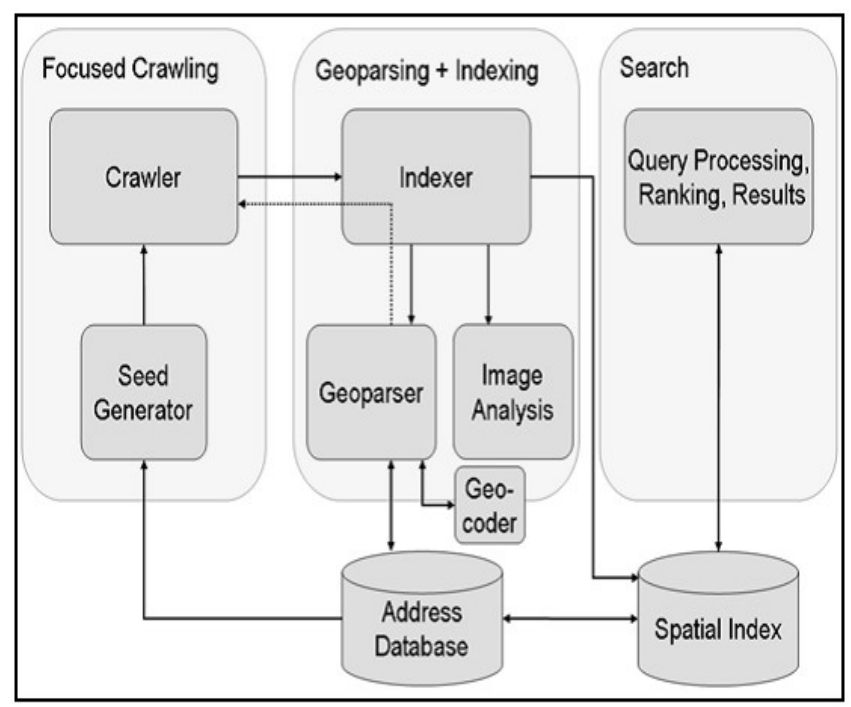

Fig. 1. Spatial search engine

The structural design as shown in Fig. 1 of spatial web search engine prototype mainly comprises on crawling, indexing and search.

\section{LITERATURE REVIEW}

Images are used as data in the area of data mining. Current research work in the field of image mining and various techniques of this field has also been summarized by Dey et al. [3]. Image segmentation is the key process in the domain of image processing. Rough set based image segmentation approach has been discussed by Roy et al. [4], it provides satisfactory method for the segmentation of image. Rough set theory is an approach to deal with vagueness and uncertainty. Chowdhuri et al. [5] presented a review for Rough set based Ad Hoc network. Karaa et al. [6] proposed a novel approach for document clustering MEDLINE based on genetic algorithm. Kausar et al. [7] discussed the data mining method applied in the extraction of clinical attribute and classification for diagnosis of cardiovascular patients. In [8] Panchal and Tiwari discussed, analyzed and compared various techniques of image retrieval system based on contents. Some features extraction techniques also described for extraction primitive features like colour, shape and texture. Combinations of these three low level features are also described. Three approaches namely Genetic Algorithm (GA), an Energy Based Model (EBM), and a Binary Integer Programming (BIP), to utilizing spatial contextual information at object level for semantic image analysis are discussed by Papadopoulos et al. [9].

A framework based on a number of various combinations of primitive features and algorithms of classification was developed for the gain of good insight on the use of spatial context. Choudhary et al. [10] described the integration of three methods that are Gabor Filter, Spatiogram, and Edge Histogram as a new method. This methodology fundamentally automates the process of retrieving by utilizing image analysis techniques taking into account primitive visual features such as texture, shape and colour with spatial information. Detailed review of various methods has been focused by Priyatharshini et al. [11]. Evaluation techniques of such methods used in the current research based on spatial features of image in content based image retrieval systems. In [12] Balasubramani and Kannan discussed about two powerful features of CBIR systems. 1. Edge Histogram Descriptor used for edge distribution 2. Spatial distribution of colours represents by Colour Layout Descriptor (CLD) in an image. More user friendly prototype NWCBIR has been developed and implemented both EHD and CLD features. Various concepts and applications of CBIR have been reviewed by Hirwane [13]. He also presented a technique on the basis of automatically derived primitive features for retrieval of images.

Jiu et al. [14] proposed a method to integrate the spatial layout and presented an application to parts classification for the estimation of human pose. Secondly author introduced edge feature as a complement from RGB images used for body parts classification. A method based on a probabilistic spatial context model, as well as individual material detection algorithm was proposed by Singhal et al. [15] to determine the scene contents. Spatial context aware material detection method used to reduce misclassification and increase the accuracy of initial classification. Construction of the surface layout of objects, aging a label to the image into geometric classes is discussed by Hoiem et al. [16]. Multiple segmentation frameworks were used to provide robust spatial support to contribute to the confidence in each geometrical label. Yadav et al. [17] presented a study of the behaviour of several content based image retrieval systems. Various feature extraction texture analysis with representation was also presented.

Shaikh et al. [18] proposed a dual layer model SHODHANI, which integrates features and processes and combine neural network approach to sense a specific image and easily retrieved from complex database. Chakraborty et al. [19] firstly estimated camera view point and position of the peoples in 3D scene then extraction spatial information from 3D people position. Neural network based a novel approach proposed by Khan et al. [20] which contributes a novel technical concept for the scene understanding and recognition, which can be important to provide vision intelligence to cloud robot.

The scope of application of intelligent software agents in the domain of web personalization for solving various currently available problems such as user profile learning, management of personalized content adaptive and modeling interactions with users has been described by Singh et al. [21]. PCA is a mathematical approach; utilization of principal component analysis in medical image processing has been discussed by Nandi et al. [22]. Generally PCA used for image fusion, feature extraction, image segmentation, compression, de-noising, registration etc. 


\section{PROBLEM DOMAIN}

One commonly asked question when confronted with a photograph is "Where is this place?" When talking about a place mentioned on the Web, the question arises "What does this place look like?” Today, these questions cannot reliable be responded in due order regarding Web pictures as they normally don't unequivocally uncover their relationship to a real geographic position. Analysis of the keywords surrounding the images or the content of the images alone has not yet achieved results that would allow deriving precise location information to select representative images. Photos that are reliably tagged with labels of place names or areas only cover a small fraction of available images and also remain at a keyword level. In this research work representative photographic representations of the mentioned location has been identified. The potential propagation of this location to the images embedded in the page and its reliability is determined by content-based and context-based analysis. In a first step, an analysis of the Web pages identifies photographs, i.e., the image content relevant for a spatial image search for realistic depictions of places. After the identification of photograph, image context and content evaluation can be performed using a location-relevance classification process to determine if the image is originally connected with the location previously recognized for the webpage.

\section{MethodOLOGY}

Proposed method for the assessment of image location based on processing chains identifies a probable locationrelation of images from website pages. This process is outlined in Fig. 2. The starting step is a web page which is examined all references of image are extracted. After this image is processed and analyzed using a filter chain in the component of photo classification. This component analyzes each and every image and decides whether it meets the requirement to be considered as photo. When condition is satisfied then adjacent page and features of image are given as input for the classification of location.

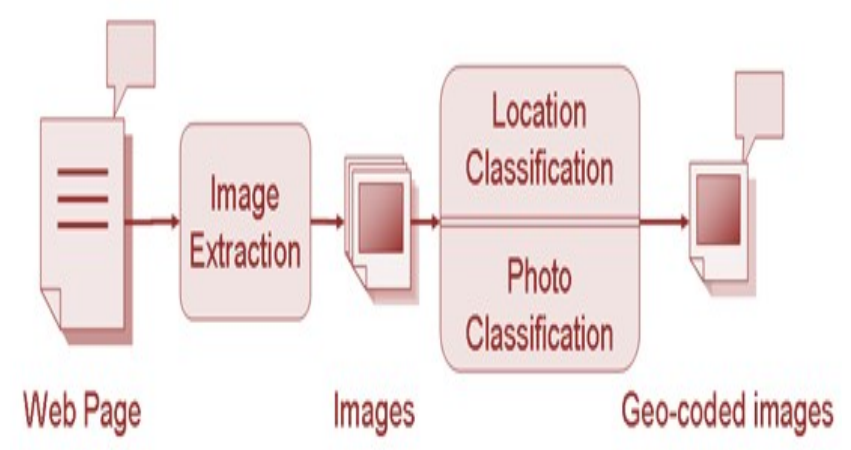

Fig. 2. Geo navigation process

For both location classification and photograph classification different sources of information has been utilized.

\section{A. Photograph classification}

Various types of images are used on web pages for different reasons. First huge amount of images are used as illustrative purpose such as, graphics, photographs, sketches, diagrams, logos, title bars. Second group of images like, bullets menu items, borders, headings, buttons etc. serves as formatting objects. Java applets flash and videos are not considered into this research work because they have different semantics as compared to images. This research study only focuses on photographs being the key possessor of the visual location information and serve illustrative purposes.

Therefore, proposed method identifies images with a high likelihood to be a reasonable photograph of a place. Various features and their relativity for photograph classification are, image size and ratio, size comparison, filename comparison, color histogram analysis, animation, EXIF data, vertical edges, face detection etc. These features are playing very important role for the classification of photographs.

\section{B. Location classification}

After photograph classification proposed method has to make connection to the location of the web page images. A picture identified with an area will display certain qualities which exploit to make an arrangement of estimations of how well the area of a Web page can be acquired by the pictures embedded in it. For the execution of the location classification, distance of the picture has been considered to every location on the page and the matching of picture descriptors and key components on the page to parts of the location. An in-page location keyword seek has been utilized.

The examined image tag attributes are name, alt and title, the set of evaluation features are image-attributes, DOMDistance, Source-Path, Page title and metadata are extremely helpful in location classification.

\section{Fused features for location assessment}

The procedure appeared before in Fig. 2 portrays two legitimate blocks for location assessment that are location classification and photograph classification. Inside every chain, a few channels serially assess the previously stated features, compute a relevance score, and include an annotation for the assessed picture. Relevance score for img per chain is as follows:

$$
\begin{gathered}
\text { relevance }(\mathrm{img})_{\text {chain }}=\sum_{i=1}^{n} w_{i} \text { evaluation }_{i}(\text { img }) \\
\text { veto }(\mathrm{img})=\exists i \in \text { o..n }: \text { evaluation }_{i}(\mathrm{img})=\text { veto }
\end{gathered}
$$

For geo location the overall accuracy of classifier is as per exact situation of their positive or negative situation, where total accurately categorized as follows:

$$
=\frac{T P+T N}{T P+T N+F P+F N}
$$

At any geo location $(R, n)$ with that $X$ pixel, may include noise and is consider with variance $\sigma 2$ as

$$
\varepsilon(K, I) \sim N(O, \sigma 2)
$$

So Markov process as it condition probability 


$$
P\left\{X\left(S_{n}\right)=i \mid X\left(S_{k}\right)\right\}
$$

Thus

$$
\begin{gathered}
X(k, l)=\sum^{M} \sum^{M} K \sin \left(Q_{r}\right) \\
\cos \left(Q_{r}\right) \\
\alpha^{r}
\end{gathered}
$$

This proposed model assumption on the parameter as shown in Fig. 3. Further restriction on the range of the parameter is placed by examining the ability of the model.

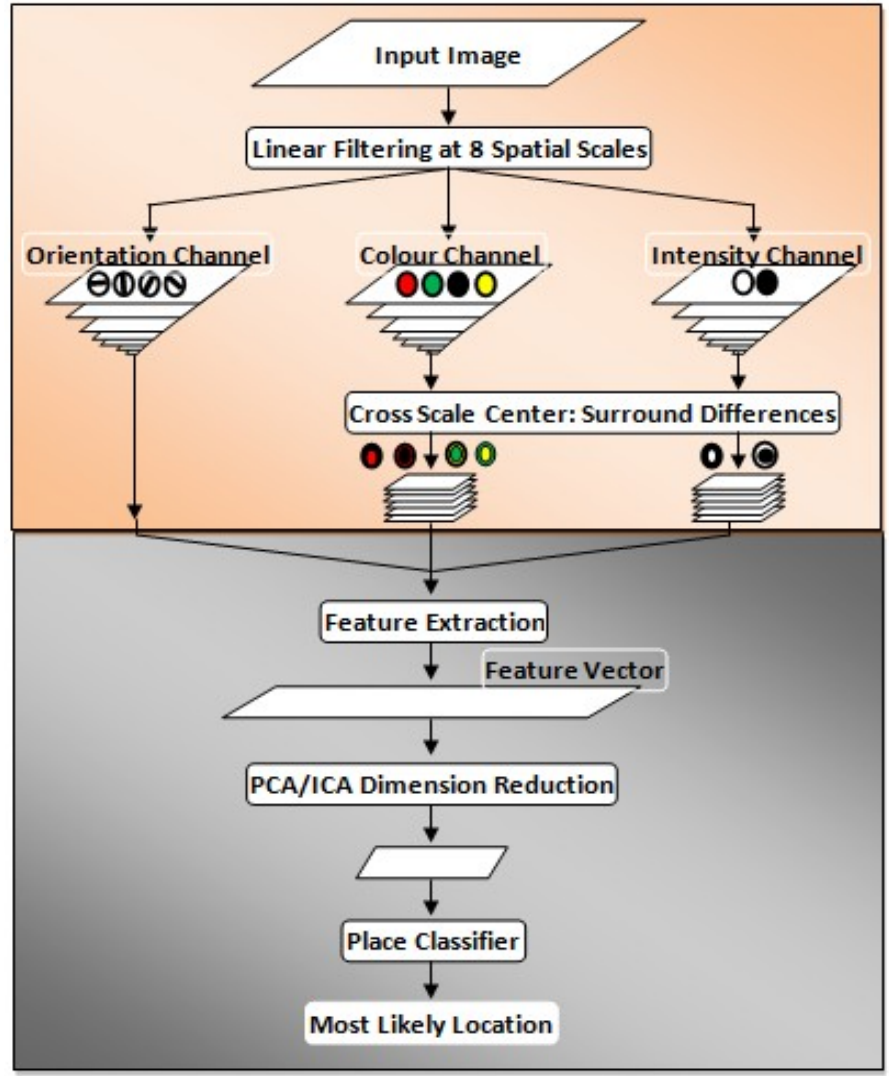

Fig. 3. Proposed process

So similarity condition prefer as per models approach vector as target image $T$, where

$$
\operatorname{Td}^{2}(x, y)=\frac{n_{x} \cdot n_{y}}{n_{x}+n_{y}}(\bar{x}-\bar{y})^{\prime} A+(\bar{x}-\bar{y})
$$

Where

$$
A=\frac{A_{x}+A_{y}}{n_{x}+n_{y}-2}
$$

So analyze query and tag image are if

$$
T^{2} \leq \frac{\left(N_{x}+N_{y}-2\right) P}{N_{x}+N_{y}-P-1}
$$

Where P size.

\section{Results and discussions}

Series of test crawls on proposed system has been preformed to evaluate and assess the validity of proposed approach. Crawl of Rugen (Germany's island) and Oldenburg (Germany's city) has been chosen as a test set. The evaluation of performance has been conducted on crawl of Oldenburg, number of URLs 17250, image references extracted by system 730845. Of these 540825 (74\%) has received veto, further 182712 (25\%) were vetoed afterwards. Remaining 7308 (1\%) received an evaluation score. The evaluation of proposed system demonstrates that the proposed approach has been efficient.

\section{CONCLUSIONS AND FUTURE WORK}

An automatic method for geo-reference images embedded in webpage on the address level has been proposed in this study. On the basis of location-based search, proposed method and heuristics to propagate the Web page location and assign it to embedded photographs. Proposed approach demonstrates that a fused multi-source analysis of context and content is suitable for this task. On unstructured web pages automatic location identification of pictures now permits pictures to become part of the results of a geospatial Web seek without past manual tagging. The results of proposed methods are promising regarding classification and performance. The present methodology is fairly exploratory, in the future work the outcomes could be made all the more soundly established by comparing diverse classification outcomes by, such as, SVM, neural networks, decision trees and so forth. This could be bolstered by the utilization of more page and features of image for the purpose of classification. The location of a page is as of now known, different sources could be utilized to demonstrate the kind of zone this area is in. Such data could be utilized to authenticate discovered images against a desire for, e.g., urban buildings, landscapes, or rural areas.

\section{ACKNOWLEDGMENT}

This paper was supported by the National Natural Science Foundation of China (Grant No.61370073), the National High Technology Research and Development Program of China (Grant No.2007AA01Z423).

\section{REFERENCES}

[1] D. Ahlers and S. Boll. Location-basedWeb search. In A. Scharl and K. Tochterman, editors, The Geospatial Web. How Geo-Browsers, Social Software and the Web 2.0 are Shaping the Network Society. Springer, London, 2007.

[2] D. Ahlers and S. Boll. Retrieving Address-based Locations from the Web. In GIR '08: 5th Workshop on Geographic Information Retrieval, pages 27-34, New York, NY, USA, 2008. ACM.

[3] Dey N, Karaa WBA, Chakraborty S, Banerjee S, Salem MAM, Azar AT (2015) Image Mining Framework and Techniques: A Review. International Journal of Image Mining, Indersceince, 1(1): 45-64.

[4] Roy P., Goswami S., Chakraborty S, Azar AT, Dey N (2014) Image Segmentation Using Rough Set Theory: A Review. International Journal of Rough Sets and Data Analysis, 1(2): 62-74. 
[5] Chowdhuri S, Roy P, Goswami S, Azar AT, Dey N (2014) Rough Set Based Ad Hoc Network: A Review. International Journal of Service Science, Management, Engineering, and Technology. 5(4): 66--76.

[6] Karaa, Wahiba Ben Abdessalem, et al. "MEDLINE Text Mining: An Enhancement Genetic Algorithm Based Approach for Document Clustering." Applications of Intelligent Optimization in Biology and Medicine. Springer International Publishing, 2016. 267-287.

[7] Kausar, Noreen, et al. "Systematic Analysis of Applied Data Mining Based Optimization Algorithms in Clinical Attribute Extraction and Classification for Diagnosis of Cardiac Patients." Applications of Intelligent Optimization in Biology and Medicine. Springer International Publishing, 2016. 217-231.

[8] Panchal, C., K., Tiwari, R., A., “A Survey on CBIR using Low Level Feature Combination”, International Journal of Emerging Technology and Advanced Engineering, Vol. 4, Issue 11, pp. 211-220, Nov. 2014.

[9] Papadopoulos, G., T., Saathoff, C., Escalante, H., J., Mezaris, V., Kompatsiaris, I., Strintzis, M., G., "A Comparative Study of Object Level Spatial Context Techniques for Semantic Analysis”, Computer Vision and Understanding, pp. 1-22, 2011.

[10] Choudhary, K., Pundlik, M., Choukse, D., “An Integrated Approach for Image Retrieval Based on Content”, International Journal of Computer Science Issues, Vol. 7, Issue 3, No. 7, pp. 42-47, May 2010.

[11] Priyatharshini, R., Surendernath, S., P., Chitrakala, S., "Applications of Spatial Features in CBIR: A Survey", International Conference on Computer Science, Engineering and Applications, pp. 303-311, 2013.

[12] Balasubramani, R., Kannan, V., "Efficient use of MPEG-7 Colour Layout and Edge Histogram Descriptor in CBIR Systems", Global Journal of Computer Sci. and Tech. Vol. 9(5), pp. 157-163, 2009.

[13] Hirwani, R., "Fundamental of Content Based Image Retrieval", International Journal of Computer Science and Technology, Vol. 3, Issue 1, pp. 114-116, 2012.
[14] Jiu, M., Wolf, C., Baskurt, A., "Integrating Spatial Layout of Object Parts into Classification without Pair wise Terms: Application to Fast Body Parts Estimation from Depth Images”, LIRIS, March 2013.

[15] Singhal, A., Luo, J., Zhu, W., "Probabilistic Spatial Context Models for Scene Content Understanding", Computer Society Conference on Computer Vision and Pattern Recognition”, Vol. 1, pp. 235-241, 2003.

[16] Hoiem, D., Efros, A., A., Hebert M., "Recovering Surface Layout from an Image”, International Journal of Computer Vision, Vol. 75(1), pp. 151-172, 2007.

[17] Yadav, A., K., Vaishali, R., R., Kumar, A., P., "Survey on Content Based Image Retrieval and Texture Analysis with Applications", International Journal of Signal Processing, Image Processing and Pattern Recognition, Vol. 7, No. 6, pp. 41-50, 2014.

[18] Shaikh, R., A., Jian-Ping, L., Khan, A., Deep, S., Kumar, K., Memon, I., "Contemporary Integration of Content Based Image Retrieval", IEEE 11th International Computer Conference on Wavelet Active Media Technology and Information Processing, Chengdu, China, pp. 301-304, 2014.

[19] Chakarborty, I., Cheng, H., Javed, O., "3D Visual Proxemics: Recognizing Human Interactions in 3D from a Single Image”, IEEE Conference on Computer Vision and Pattern Recognition, pp. 34063413, 2013.

[20] Khan, A., Jian-Ping, L., Shaikh, R., A., Deep, S., Kumar, K., Hasan, F., "Vision Prehension with CBIR for Cloud Robo", 11th International Computer Conference on Wavelet Active Media Technology and Information Processing, China, pp. 293-296, 2014.

[21] Singh, Aarti, Anu Sharma, and Nilanjan Dey. "Semantics and Agents Oriented Web Personalization: State of the Art." International Journal of Service Science, Management, Engineering, and Technology (IJSSMET) 6.2 (2015): 35-49.

[22] Nandi, Dibyadeep, et al. "Principal component analysis in medical image processing: a study." International Journal of Image Mining 1.1 (2015): 65-86. 